\title{
Protease-Activated Receptor 1 Enhances Poly l:C Induction of the Antiviral Response in Macrophages and Mice
}

\author{
Silvio Antoniak ${ }^{\mathrm{a}, \mathrm{b}}$ Kohei Tatsumi ${ }^{\mathrm{a}}$ Michael Bode $^{\mathrm{a}}$ Swetha Vanja ${ }^{\mathrm{a}}$ \\ Julie C. Williams ${ }^{\text {a }}$ Nigel Mackman $^{\text {a }}$ \\ aThrombosis and Hemostasis Program, Division of Hematology and Oncology, Department of Medicine, \\ UNC McAllister Heart Institute and b ${ }^{b}$ Department of Pathology and Laboratory Medicine, University of \\ North Carolina at Chapel Hill, Chapel Hill, N.C., USA
}

\section{Key Words}

Protease-activated receptor 1 - Double-stranded RNA.

Innate immune response $\cdot$ Spleen · Macrophage

\section{Abstract}

The coagulation cascade is activated during viral infections as part of the host defense system. Coagulation proteases activate cells by cleavage of protease-activated receptors (PARs). Recently, we reported that the activation of PAR-1 enhanced interferon (IFN) $\beta$ and CXCL10 expression in cardiac fibroblasts and in the hearts of mice infected with Coxsackievirus B3. In this study, we used the double-stranded RNA mimetic polyinosinic:polycytidylic acid (poly I:C) to induce an antiviral response in macrophages and mice. Activation of PAR-1 enhanced poly I:C induction of IFN $\beta$ and CXCL10 expression in the murine macrophage cell line RAW264.7, bone-marrow derived mouse macrophages (BMM) and mouse splenocytes. Next, poly I:C was used to induce a type I IFN innate immune response in the spleen and plasma of wild-type (WT) and PAR-1-1- mice. We found that poly I:C treated PAR-1 ${ }^{-/-}$mice and WT mice given the thrombin inhibitor dabigatran etexilate exhibited signifi-

\section{KARGER}

(C) 2016 S. Karger AG, Basel cantly less IFN $\beta$ and CXCL10 expression in the spleen and plasma than WT mice. These studies suggest that thrombin activation of PAR-1 contributes to the antiviral response in mice.

(c) 2016 S. Karger AG, Basel

\section{Introduction}

Interferons (IFNs) are divided into 3 groups: type I $(\operatorname{IFN} \alpha / \beta)$, type II (IFN $\gamma)$ and type III (IFN $\lambda)$. Most cells express type I IFN $\alpha / \beta$. In contrast, IFN $\gamma$ synthesis is restricted to activated T cells and natural killer (NK) cells, and acts to promote the transition from the innate to the adaptive immune response [1]. Synthesis of IFNs is an essential part of antiviral innate immune responses [1]. Indeed, a deficiency of IFNs or IFN-dependent signaling is associated with increased susceptibility to certain virus infections, such as enteroviruses, influenza and herpes virus [1].

Double-stranded RNA (dsRNA) is a potent trigger of the type I IFN response [1]. dsRNA is part of the genome of dsRNA viruses and is an intermediate of single-strand 
RNA virus replication [1]. dsRNA is detected by the transmembrane receptor toll-like receptor (TLR) 3 , as well as the cytosolic receptors dsRNA-dependent protein kinase, retinoic acid-inducible gene I and melanoma differentiation-associated gene $5[2,3]$. The dsRNA mimetic polyinosinic:polycytidylic acid (poly I:C) is used experimentally to stimulate TLR3 and induce the expression of type I IFNs in vitro and in vivo [1,3-5].

TLR3 is broadly expressed by myeloid and nonmyeloid cells $[3,6]$. Within the spleen, high levels of TLR3 expression are present in the cells of the red pulp as well as in the marginal zone, while lower levels of expression are present in macrophages and dendritic cells (DCs) in the white pulp [6]. TLR3-dependent innate immune activation is essential to fight particular virus infections, including poliovirus, rhinovirus, herpes simplex, encephalomyocarditis and Coxsackievirus group B (CVB) [3]. In addition, TLR3 enhances the antiviral responses of macrophages against other viruses, such as the monkey and human immunodeficiency viruses (HIV) $[7,8]$.

Virus infections activate the coagulation system by inducing tissue factor (TF) expression in various cell types [9-11]. Interestingly, one study found that poly I:C induced TF expression in endothelial cells but not monocytes [12]. Furthermore, administration of poly I:C to mice increased systemic activation of coagulation and fibrin deposition in the liver in a TLR3-dependent manner [12]. Thrombin is the central protease of the coagulation cascade but also activates cells by cleavage of proteaseactivated receptors (PARs), such as PAR-1 [13]. This receptor is also activated by other proteases, such as matrix metalloproteinases (MMP) 1 and MMP13 [4, 9, 10, 14, 15]. PAR-1 is expressed by a variety of human and murine immune cells, including monocytes/macrophages, DCs, T cells and NK cells $[16,17]$.

The coagulation and immune systems appear to have a common evolutionary origin [18]. Moreover, there is crosstalk between these two systems during infections [9, $10,17,18]$. It has been proposed that TLRs and PARs act as a cooperative sensor system to detect pathogens $[10$, 19]. We demonstrated that PAR-1 activation enhanced poly I:C-induced IFN $\beta$ and CXCL10 expression in murine cardiac fibroblasts, suggesting an interaction between PAR-1 and TLR3 [4]. In a CVB3 mouse model, we observed that a deficiency of PAR-1 was associated with reduced type I IFN responses, leading to increased myocarditis [4]. In addition, inhibition of the PAR-1 activating proteases thrombin or MMP13 led to increased myocarditis [4]. Bone marrow transplantation experiments further revealed that PAR-1 on both nonhematopoietic and hematopoietic cells contributed to PAR-1 mediated protection against CVB3 myocarditis [4]. This finding is consistent with the observation that cardiac damage and survival of CVB4-infected TLR3-deficient mice can be improved by the adaptive transfer of wild-type (WT) macrophages [20].

In this study, we utilized poly I:C as a surrogate for viral activation and $\mathrm{PAR}-1^{-1-}$ mice to investigate the role of PAR-1 in the induction of an antiviral innate immune response in macrophages and mice.

\section{Material and Methods}

Mice

We used WT and PAR-1 ${ }^{-/-}$mice on a C57BL/6J background [21]. All mouse studies were performed with the approval of the University of North Carolina at Chapel Hill Institutional Animal Care and Use Committee.

\section{Isolation and in vitro Stimulation of Macrophages, DCs and}

Splenocytes

WT and PAR- $1^{-1-}$ mice (4 mice each, aged 8-12 weeks) were used to isolate splenocytes or generate bone marrow-derived macrophages and dendritic cells (BMMs and BMDCs). Each isolation was repeated once. For splenocyte isolation, spleens were harvest and passed through a $70-\mu \mathrm{m}$ cell strainer to generate a single-cell suspension. Red blood cells were lysed and the splenocytes washed and resuspended at a density of $1 \times 10^{6}$ cells per well of a 24 -well plate in RPMI 1640 with 10\% FBS and 1\% penicillin/streptomycin. Cells were stimulated with $25 \mu \mathrm{g} / \mathrm{ml}$ poly I:C ( $\gamma$-radiated; SigmaAldrich, St. Louis, Mo., USA) and/or PAR-1 agonist peptide (TFLLR-NH $\mathrm{N}_{2}, 200 \mu \mathrm{M}$, Abgent, San Diego, Calif., USA) for $2 \mathrm{~h}$.

BMMs were generated from bone marrow using the L929-conditioned media method as described [22]. BMDCs were derived from bone marrow using GM-CSF and IL-4 (30 ng/ml each) as described [23]. RAW264.7 cells, a murine macrophage cell line, were maintained as described [24].

For experiments, BMMs were plated at a density of $1 \times 10^{6}$ cells per well of a 12-well plate the day before stimulation. The next day, cell culture media was replaced with $0.5 \mathrm{ml}$ fetal bovine serum (FBS)-free media and the cells were stimulated with poly I:C (25 $\mu \mathrm{g} / \mathrm{ml})$ and/or PAR-1 agonist peptide $(200 \mu \mathrm{M})$ for different times. BMDCs were seeded in FBS-containing culture media at a density of $1 \times 10^{6}$ cells in $1 \mathrm{ml}$ of media the day before stimulation. The next day, cells were stimulated without changing the media with poly I:C $(25 \mu \mathrm{g} / \mathrm{ml})$ and/or PAR-1 agonist peptide $(200 \mu \mathrm{M})$ for $8 \mathrm{~h}$. RAW264.7 cells were seeded at a density of $5 \times 10^{5}$ cells per well of a 12 -well plate 1 day prior to stimulation. Media was replaced with $0.5 \mathrm{ml} \mathrm{FBS}$-free culture media the next day and the cells were stimulated with $5 \mu \mathrm{g} / \mathrm{ml}$ poly I:C and/or $150 \mu \mathrm{M}$ PAR-1 agonist peptide for the indicated times.

For measuring IFN $\beta$ promoter activity, RAW264.7 cells were stable-transfected with the pNiFty2-IFB-SEAP plasmid (containing the Ifnb1 promoter) with Zeocin selection (Invivogen, San Diego, Calif., USA) using Lipofectamine 2000 (Life Technologies, Carlsbad, Calif., USA) [4]. Cells were seeded and treated with poly 
Fig. 1. Effect of PAR-1 activation and deficiency on poly I:C induction of gene expression in macrophages and splenocytes. a IFN $\beta$ promoter activity measured by SEAP release in the media from pNiFty2IFB-SEAP plasmid expressing RAW264.7 macrophages stimulated with poly I:C $(5 \mu \mathrm{g} / \mathrm{ml})$ with or without $5 \mathrm{nM}$ thrombin (Thr) for $24 \mathrm{~h}$. IFN $\beta$ (b) and CXCL10 (c) mRNA expression in RAW264.7 macrophages stimulated with poly I:C $(5 \mu \mathrm{g} / \mathrm{ml})$ and/or $150 \mu \mathrm{M}$ PAR-1 agonist peptide (AP) for $2 \mathrm{~h}$. Levels of mRNA are shown relative to poly I:C-stimulated cells which were set to 1. d Levels of CXCL10 in the cell culture media of RAW264.7 macrophages with or without poly I:C $(5 \mu \mathrm{g} / \mathrm{ml})$ with or without PAR-1 AP $(150 \mu \mathrm{M})$ for $7 \mathrm{~h}$. e IFN $\beta$ mRNA expression in WT (white bars) and PAR$1^{-l-}$ (black bars) BMMs before and after stimulation with poly I:C $(25 \mu \mathrm{g} / \mathrm{ml})$ with or without PAR-1 AP $(200 \mu \mathrm{M})$ for $2 \mathrm{~h}$. Results are shown relative to poly I:C-stimulated WT splenocytes or BMMs which were set to 1 . f Levels of CXCL10 in the cell culture media of WT (white bars) and PAR-1 ${ }^{-/-}$(black bars) BMMs before and after stimulation with or without poly I:C (25 $\mu \mathrm{g} / \mathrm{ml})$ with or without PAR-1 AP $(200 \mu \mathrm{M})$ for $8 \mathrm{~h}$. IFN $\beta$ (g) and CXCL1 (h) mRNA expression in WT (white bars) and PAR$1^{-/-}$(black bars) splenocytes before and after stimulation with poly I:C $(25 \mu \mathrm{g} / \mathrm{ml})$ with or without PAR-1 AP $(200 \mu \mathrm{M})$ for $2 \mathrm{~h}$. Results are shown relative to poly I:Cstimulated WT splenocytes or BMMs which were set to 1 . Data were normalized to GAPDH mRNA levels. Data (mean \pm SEM; $\mathrm{n}>4$ ) presented as arbitrary units $(\mathrm{AU})$ if not stated otherwise were analyzed by 1-way (a-d) and 2-way (e-h) ANOVA. Statistical significance is shown as $*$ p $<$ $0.05,{ }^{\#} \mathrm{p}<0.05$ versus unstimulated control within the same genotype or unstimulated control, and ${ }^{\$} \mathrm{p}<0.05$ versus poly I:C alone.
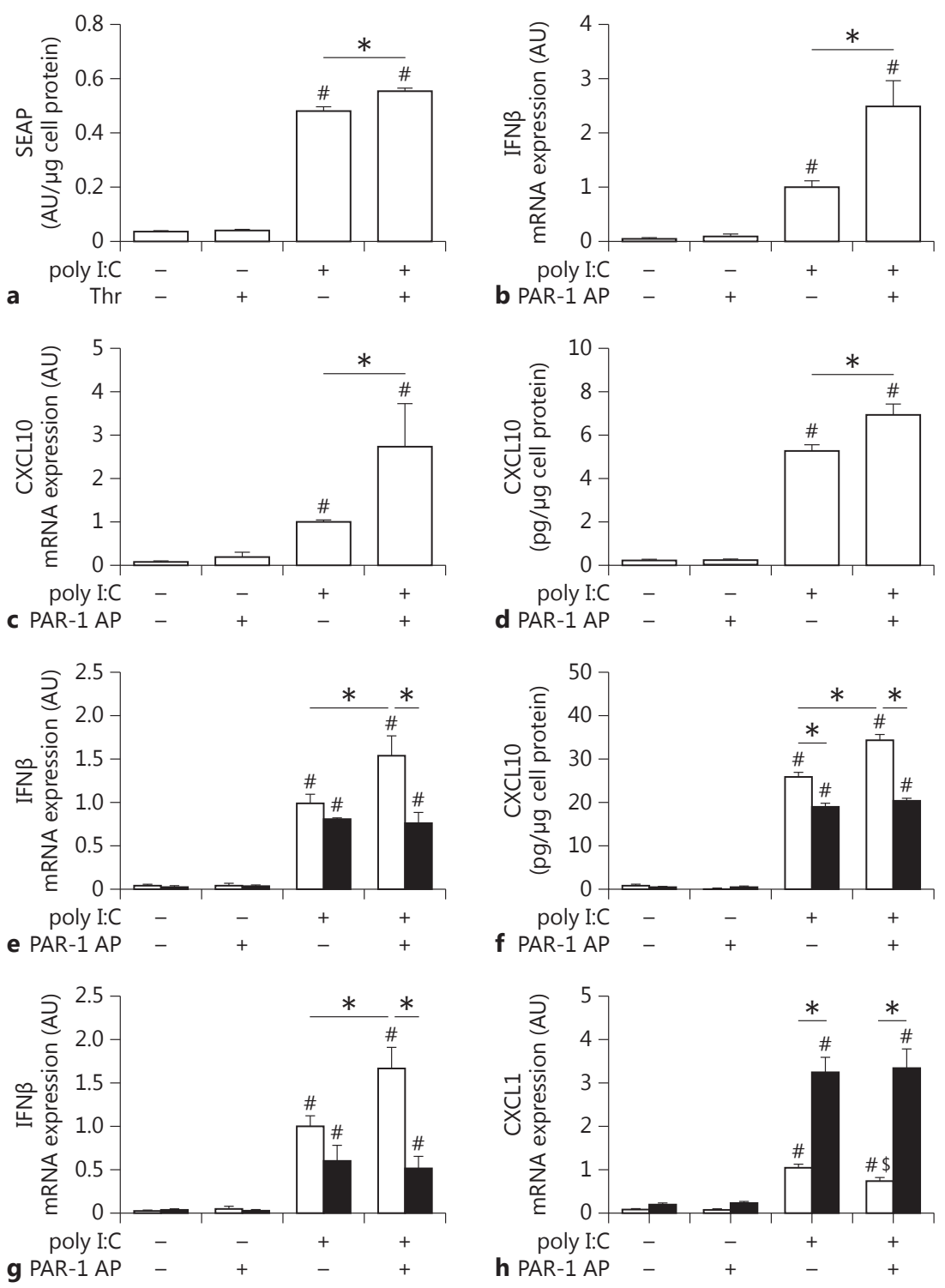

$\mathrm{I}: \mathrm{C}(5 \mu \mathrm{g} / \mathrm{ml})$ and/or $5 \mathrm{nM}$ of thrombin (human $\alpha$-thrombin; Enzyme Research Laboratories, South Bend, Ind., USA) as mentioned above. The next day, media was collected, and SEAP (secreted embryonic alkaline phosphatase) activity was detected using QUANTI-blue (Invivogen) at $640 \mathrm{~nm}$ with a SpectraMax M5 plate reader (Molecular Devices, Sunnyvale, Calif., USA) [4].

\section{In vivo Stimulation}

Male mice (8-12 weeks old) were injected intraperitoneally (i.p.) with $8 \mathrm{mg} / \mathrm{kg}$ poly I:C (Sigma-Aldrich) diluted in PBS [25]. Blood was collected from the inferior vena cava and plasma was prepared as previously described $[11,26]$.

PAR-1 and TLR3 Responses in Macrophages

\section{Thrombin Inhibition}

To inhibit thrombin, mice were fed for 7 days with a custommade chow (AIN-93M, Dyets Inc., Bethlehem, Pa., USA) with peanut flavoring ( $2 \mathrm{~g} / \mathrm{kg}$ chow) with or without dabigatran etexilate (10 g/kg chow) as described [4]. The level of anticoagulation was measured as described [4].

\section{CVB3 Infection Model}

A standard CVB3 infection model was used $[4,5,27]$. Briefly, male mice (aged 6-8 weeks) were infected with an i.p. injection of CVB3 Nancy Strain ( $10^{5}$ plaque-forming units in $200 \mu \mathrm{l}$ saline). Tissue and blood were collected and analyzed 4 days after infection. 
Real-Time PCR

Total RNA from cultured cells or splenic tissue was isolated by the TRIzol method (Life Technologies). One microgram of RNA was reverse-transcribed into cDNA using the iScript ${ }^{\mathrm{TM}} \mathrm{RT}$ Supermix (Bio-Rad Laboratories, Hercules, Calif., USA) [28, 29]. Realtime PCR primer/probes for IFN $\beta$, IFN $\gamma$, CXCL10, CCL5, CXCL1, IRF3, IRF7, viperin, Rpl4, GAPDH and $18 \mathrm{~S}$ were purchased from Integrated DNA Technologies (Coralville, Ia., USA). CVB3 genome levels were quantified as recently described [4,5]. Real-time PCR reactions were performed using the SSoFast ${ }^{\mathrm{TM}}$ Advanced Universal Supermix in a BioRad cycler (Bio-Rad Laboratories). The housekeeping genes Rpl4 or 18S (for splenic tissue) and GAPDH (for cells) were used to correct for variations in input RNA and reaction efficiency [11].

\section{ELISA}

IFN $\beta$ (PBL Assay Science, Piscataway, N.J., USA), CXCL10, CCL5 and CXCL1 (Duo-Set, R\&D Systems, Minneapolis, Minn., USA) ELISAs were used to measure levels of IFN $\beta$ and CXCL10, CCL5 and CXCL1 in plasma and/or cleared cell culture supernatant $\left(500 \mathrm{~g}, 10 \mathrm{~min}, 4^{\circ} \mathrm{C}\right)[4]$.

\section{Statistics}

All statistical analyses were performed using GraphPad Prism (v5.0; GraphPad Software Inc., La Jolla, Calif., USA). Data are represented as mean \pm SEM. For 2-group comparison of continuous data, 2-tailed Student's t test was used. For multiple-group comparison, data were analyzed by 1- or 2-way ANOVA and Bonferroni's correction was used for repeated measures over time. A $\mathrm{p}$ value $<0.05$ was considered significant.

\section{Results}

\section{PAR-1 Activation Enhances Poly I:C Induction}

of IFN $\beta$ and CXCL10 Expression in Murine

Macrophages and Splenocytes

Macrophages and DCs are known to reside in the spleen and respond to TLR3 stimulation by expressing IFN $\beta$ and CXCL10 [25, 30, 31]. In addition, depletion of phagocytic splenic macrophages and DCs of the marginal zone markedly reduce antiviral responses [32]. We analyzed the role of PAR- 1 in the poly I:C induction of IFN $\beta$ and CXCL10 expression in the murine macrophage cell line RAW264.7, BMMs, BMDCs and splenocytes from WT and PAR- $1^{-/-}$mice.

Poly I:C induction of IFN $\beta$ promoter activity and IFN $\beta$ and CXCL10 mRNA and protein expression in RAW264.7 cells were significantly enhanced by PAR-1 activation (fig. 1a-d). Next, we analyzed poly I:C induction of IFN $\beta$ mRNA expression and CXCL10 protein expression in BMMs derived from WT and PAR-1 ${ }^{-/-}$mice. Poly I:C alone induced IFN $\beta$ mRNA expression in both WT and PAR-1 ${ }^{-1-}$ BMMs and splenocytes, but the PAR-1 agonist peptide alone had no effect (fig. 1e, g). Impor- tantly, costimulation of cells with poly I:C and the PAR-1 agonist peptide significantly increased IFN $\beta$ mRNA expression in WT BMMs and splenocytes but not in PAR$1^{-/-}$BMMs and splenocytes (fig. 1e, g). In addition, poly $\mathrm{I}: \mathrm{C}$ stimulation of $\mathrm{WT}$ and $\mathrm{PAR}-1^{-1-} \mathrm{BMMs}$ induced CXCL10 expression, which was significantly increased in WT BMMs but not PAR-1 $1^{-/-}$BMMs costimulated with PAR-1 agonist peptide (fig. 1f). We did not detect any CXCL10 expression in splenocytes after $2 \mathrm{~h}$ of poly I:C stimulation.

Poly I:C-stimulated PAR-1 $1^{-/-}$BMMs and BMDCs expressed lower levels of CXCL10 than WT BMMs and BMDCs (fig. 1f; data not shown). This result is consistent with previous studies showing that the poly I:C stimulation of cardiac fibroblasts induces the expression of MMP13 that activates PAR-1 $[4,10]$. Similar results were observed for CXCL10 protein expression $(8 \mathrm{~h})$ with WT and PAR- $1^{-/-}$BMDCs (data not shown).

Recently, we observed increased levels of CXCL1 in the lungs of influenza A virus-infected PAR-1 $1^{-/}$mice compared to WT mice [4]. In addition, IL-8, the human homolog of murine CXCL1, is directly induced after TLR3 stimulation [33]. Therefore, we analyzed CXCL1 expression after poly I:C and/or PAR-1 stimulation in vitro. Poly I:C induced CXCL1 by splenocytes but PAR-1 stimulation alone had no effect (fig. 1h). Unexpectedly, WT splenocytes exhibited 3-fold lower levels of CXCL1 mRNA after stimulation with poly I:C compared to stimulated PAR-1 ${ }^{-/-}$splenocytes (fig. 1h). Furthermore, activation of PAR-1 in WT splenocytes but not in PAR-1 $1^{-/-}$ splenocytes significantly reduced the poly I:C induction of CXCL1 mRNA expression (fig. 1h).

\section{Poly I:C Induces an Antiviral Response in WT Mice}

Poly I:C injection in mice leads to maximum type I IFN expression for between 2 and $4 \mathrm{~h}$ in the plasma [25, 34]. In addition, poly I:C-mediated synthesis of IFN $\beta$ is followed by an increase in CXCL10 plasma levels [34]. In our mouse studies, we focused on changes in gene expression in the spleen due to the presence of blood and lymphoid elements as well as the role of poly I:C in initiating antiviral responses to blood-borne pathogens [25]. We also measured immune mediators in the plasma. To model virus-like infection of mice, WT mice were injected with poly I:C $(8 \mathrm{mg} / \mathrm{kg})$ i.p.. The expression of IFN $\beta$, IFN $\gamma$, CXCL10, CCL5, CXCL1, IRF7 and viperin mRNA increased in the spleen after the poly I:C injection (fig. 2ah). Maximal levels of these different immune mediators were observed at different times. The different gene expression pattern can be divided into early (IFN $\beta$, CXCL10 
Fig. 2. PAR-1 deficiency leads to altered splenic mRNA expression of innate immune response genes after poly I:C injection. WT mice were injected with poly I:C $(8 \mathrm{mg} / \mathrm{kg})$ and levels of $\operatorname{IFN} \beta(\mathbf{a}), \operatorname{IFN} \gamma(\mathbf{b})$, CXCL10 (c), CCL5 (d), CXCL1 (e), IRF3 (f), IRF7 (g) and viperin (h) mRNA expression were quantified by real-time PCR before and 2, 4 and $6 \mathrm{~h}$ after injection. Expression levels of controls at $0 \mathrm{~h}$ were set to 1 . i mRNA expression in spleens of WT (white bars) and PAR-1 ${ }^{-/-}$(black bars) mice $2 \mathrm{~h}$ after poly I:C injection. Expression levels of WT were set to 1. Data were normalized to Rpl4 mRNA levels. Data (mean \pm SEM; $\mathrm{n}>4$ ) presented as arbitrary units (AU) if not stated otherwise were analyzed by 1-way ANOVA (a-h) or Student's t test (i). Statistical significance is shown as ${ }^{\#} \mathrm{p}<0.05$ versus unstimulated control and $* \mathrm{p}<0.05$ versus WT.
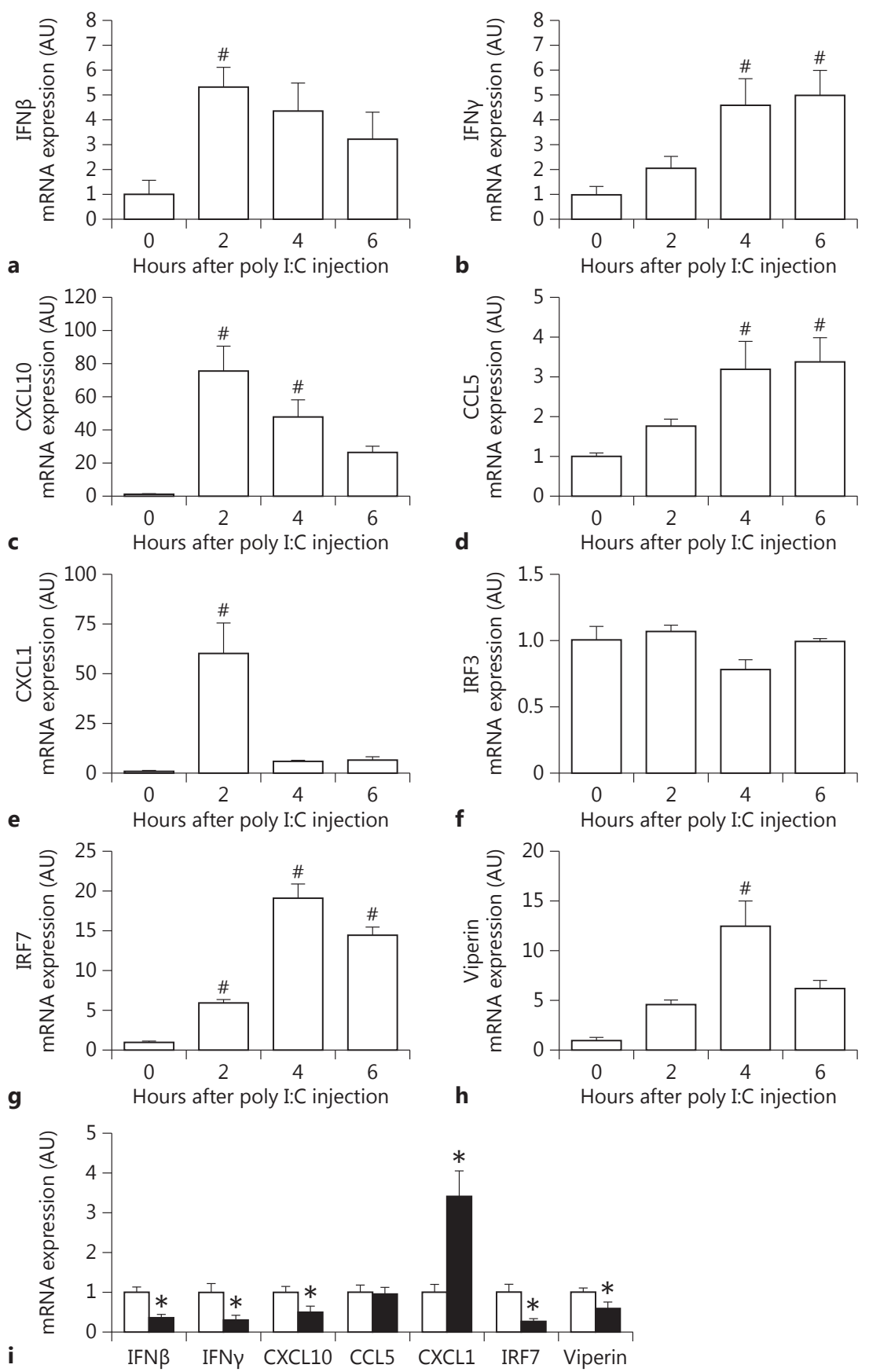

and CXCL1), intermediate (IRF7 and viperin) and late $(\mathrm{IFN} \gamma)$. However, we did not observe any changes in the splenic IRF3 mRNA expression after poly I:C injection (fig. 2f). Next, we analyzed the time-dependent changes in protein expression in the spleen. Maximal levels of
IFN $\beta$ were observed $2 \mathrm{~h}$ after the poly I:C injection (fig. 3a). Similar kinetics of IFN $\beta$ expression were observed in the plasma (fig. 4a). IFN $\beta$ induces several antiviral response genes, such as CXCL10 and CCL5 [5, 33, 34]. In addition, poly I:C induces IL-8 expression [33]. 
Fig. 3. PAR-1 deficiency leads to altered splenic protein expression of innate immune response genes after poly I:C injection. Changes in splenic protein levels of $\operatorname{IFN} \beta$ (a), CXCL10 (c), CCL5 (e) and CXCL1 ( $\mathbf{g}$ ) in WT (white bars) mice before and 2, 4 and 6 h after poly I:C injection (8 $\mathrm{mg} / \mathrm{kg}$ ). IFN $\beta$ (b), CXCL10 (d), CCL5 (f) and CXCL1 (h) protein levels in spleens of WT (white bars) and PAR-1 ${ }^{-/-}$(black bars) mice 2 and $4 \mathrm{~h}$ after poly I:C injection. Data (mean \pm SEM; $\mathrm{n}>4$ ) were analyzed by 1-way (a, c, e, g) and 2-way (b, d, f, h) ANOVA. Statistical significance is shown as ${ }^{*} \mathrm{p}<0.05$ and $^{\#} \mathrm{p}<0.05$ versus $0 \mathrm{~h}$.

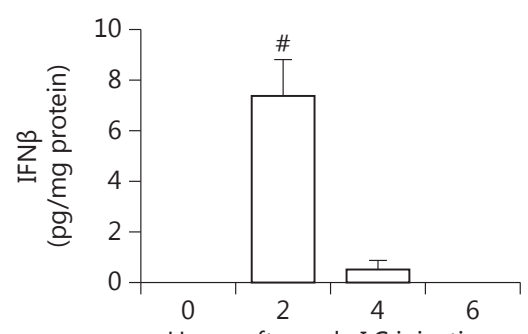

a

Hours after poly I:C injection

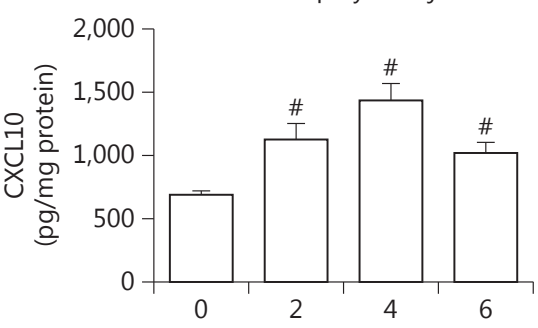

c

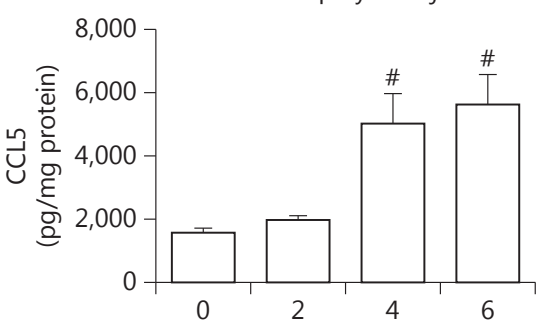

e

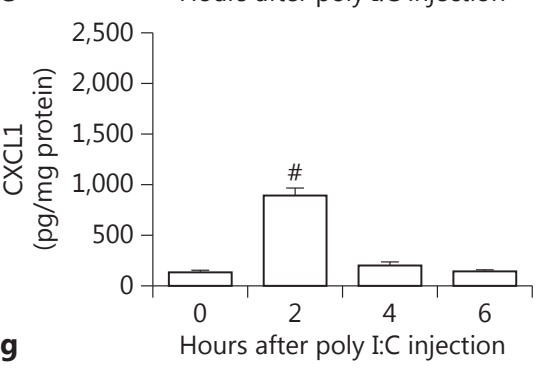

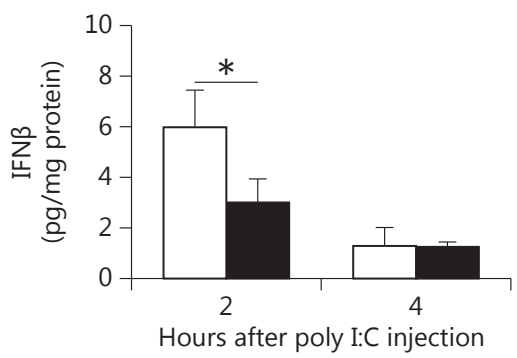

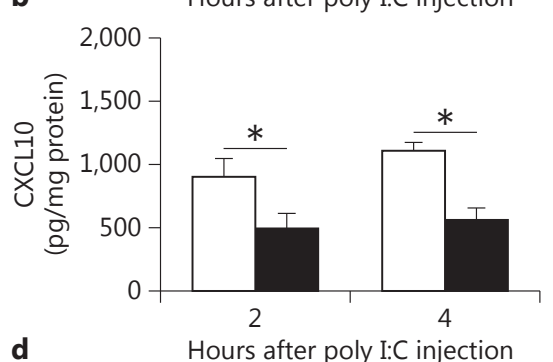

Hours after poly I:C injection

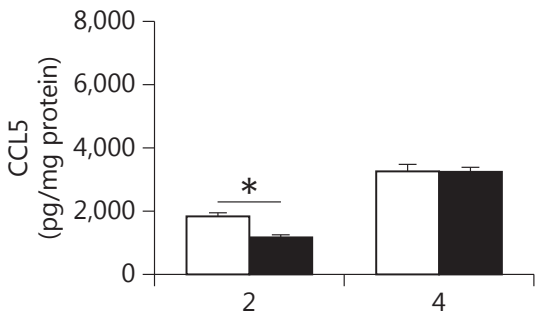

Hours after poly I:C injection

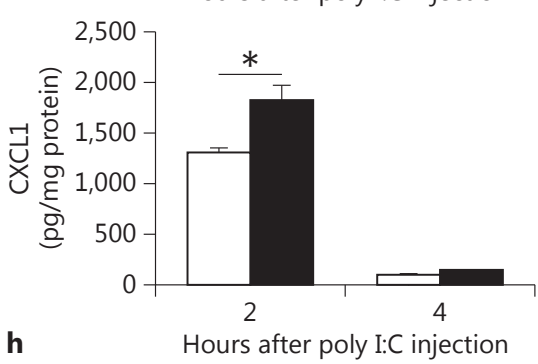

Poly I:C increased levels of CXCL10, CCL5 and CXCL1 in the spleen (fig. 3c, e, g). Maximal levels of IFN $\beta$ and CXCL1 were observed at $2 \mathrm{~h}$ whereas CXCL10 and CCL5 peaked at later times. Similar results were observed for the expression of these antiviral proteins in the plasma (fig. 4c, e, g).

\section{PAR-1 Deficiency Is Associated with Reduced Poly \\ I:C Induction of Antiviral Proteins in the Spleen and \\ Plasma}

Recently, we observed that PAR-1 expression on both nonhematopoietic and hematopoietic cells contributed to antiviral responses against the single-strand RNA virus
CVB3 in mice [4]. To determine if PAR-1 contributes to innate immune responses to poly I:C in vivo, WT and PAR- $1^{-/-}$mice were injected with poly I:C and the immune response in the spleen and plasma was analyzed. A deficiency of PAR-1 was associated with significantly lower levels of poly I:C-dependent induction of IFN $\beta$, IFN $\gamma$, CXCL10, IRF7 and viperin but not CCL5 mRNA expression (fig. 2i). In line with our in vitro data, we observed significantly higher levels of CXCL1 mRNA in PAR- $1^{-1-}$ spleens than in WT spleens (fig. 2i). In addition, $\mathrm{PAR}^{-1^{-/}}$mice injected with poly I:C had significantly lower splenic IFN $\beta$ protein levels than WT mice at $2 \mathrm{~h}$ but not $4 \mathrm{~h}$ (fig. 3b). The level of splenic CXCL10 protein in 
Fig. 4. PAR-1 deficiency leads to altered plasma protein expression after poly I:C injection. Changes in plasma protein levels of IFN $\beta$ (a), CXCL10 (c), CCL5 (e) and CXCL1 ( $\mathbf{g}$ ) in WT (white bars) mice before and 2, 4 and 6 h after poly I:C injection (8 $\mathrm{mg} / \mathrm{kg}$ ). IFN $\beta$ (b), CXCL10 (d), CCL5 (f) and CXCL1 (h) protein levels in plasma of WT (white bars) and PAR-1 ${ }^{-/-}$(black bars) mice 2 and $4 \mathrm{~h}$ after poly I:C injection. Data (mean \pm SEM; $\mathrm{n}>5$ ) were analyzed by 1-way (a, c, e, g) and 2-way (b, d, f, h) ANOVA. Statistical significance is shown as ${ }^{*} \mathrm{p}<0.05$ and $^{\#} \mathrm{p}<0.05$ versus $0 \mathrm{~h}$.
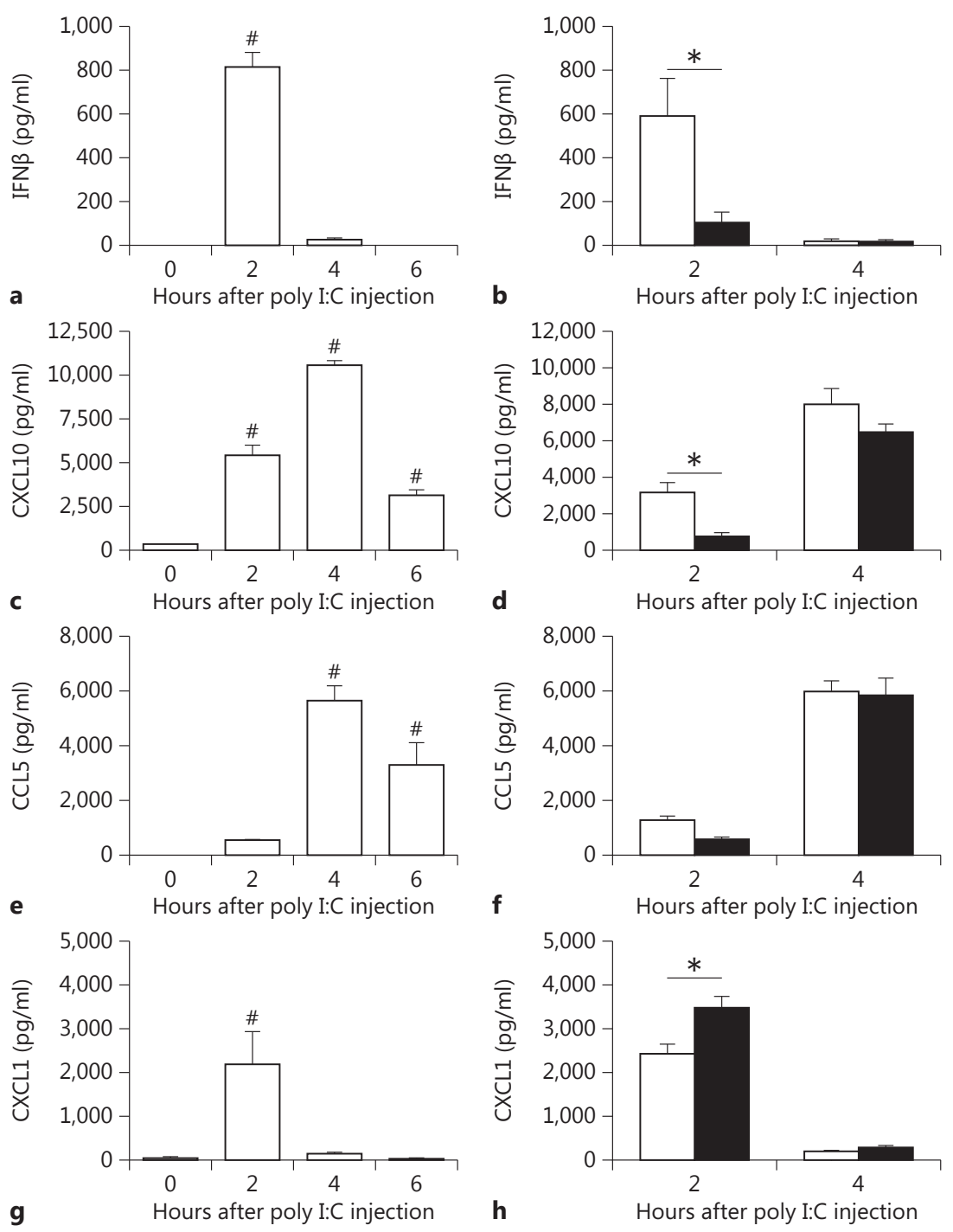
Hours after poly I:C injection
PAR-1 ${ }^{-l-}$ mice was also significantly lower at 2 and $4 \mathrm{~h}$ after poly I:C injection than in WT mice (fig. $3 \mathrm{~d}$ ). In addition, CCL5 protein levels were lower in the spleens of PAR-1 ${ }^{-1-}$ mice injected with poly I:C when compared to WT mice (fig. 3f). Consistent with the changes in splenic CXCL1 mRNA levels, CXCL1 protein levels in the spleens of PAR- ${ }^{-/-}$mice injected with poly I:C were significant higher when compared to WT mice at $2 \mathrm{~h}$ (fig. $3 \mathrm{~h}$ ).

Next, we analyzed the effect of PAR-1 deficiency on poly I:C induction of IFN $\beta$, CXCL10, CCL5 and CXCL1 in the plasma. PAR-1 $1^{-/}$mice had significantly lower levels of IFN $\beta$ and CXCL10 in the plasma $2 \mathrm{~h}$ after poly I:C treatment compared to WT mice (fig. 4b, d). CCL5 plas- ma levels were lower in PAR-1 $1^{-/-}$mice than in WT mice but this difference was not statistically significant (fig. 4f). As expected from the splenic data, the CXCL1 plasma levels were significantly higher in PAR- $1^{-/-}$mice than in WT mice $2 \mathrm{~h}$ after poly I:C injection (fig. $4 \mathrm{~h}$ ).

\section{Inhibition of Thrombin Is Associated with Reduced} Poly I:C Induction of Antiviral Proteins in the Spleen and Plasma

Thrombin is the primary activator of PAR-1 in vivo [13]. Furthermore, poly I:C injection of the mice was associated with the increased activation of coagulation [12]. We observed a maximal activation of coagulation, as mea- 


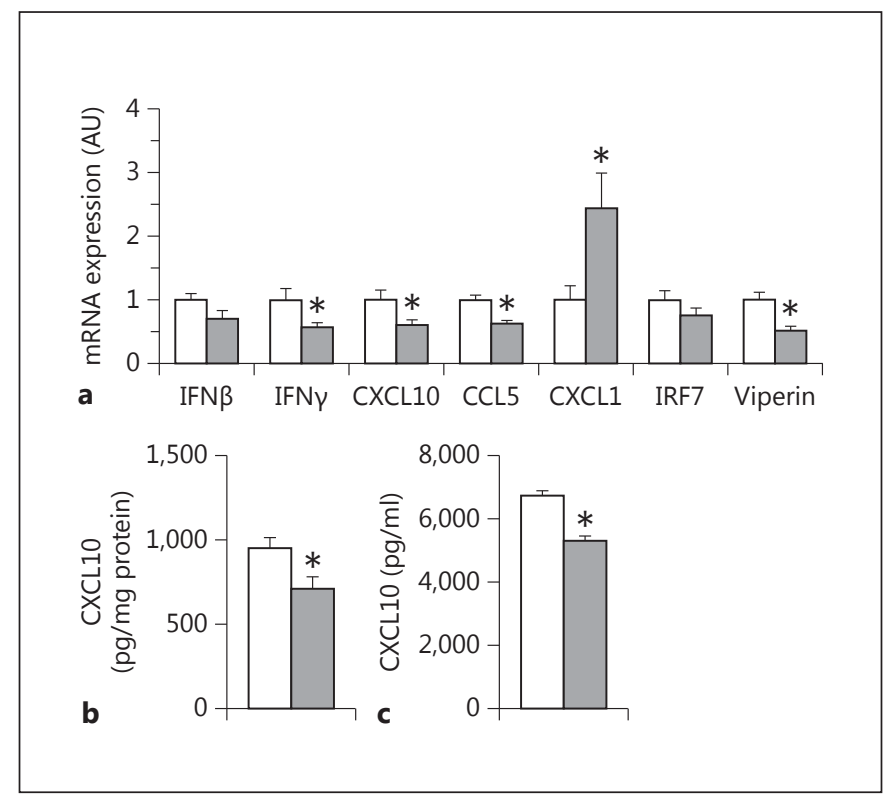

Fig. 5. Thrombin inhibition alters the immune response to poly I:C in the spleen and plasma. WT mice were treated with dabigatran etexilate (10 g/kg chow, gray bars) or placebo (white bars) for 7 days prior to poly I:C challenge ( $8 \mathrm{mg} / \mathrm{kg})$. a mRNA expression in the spleen $4 \mathrm{~h}$ after poly I:C injection. Data were normalized to Rpl4 mRNA levels. CXCL10 levels in the spleen (b) and plasma (c) $4 \mathrm{~h}$ after poly I:C injection. Data (mean \pm SEM; $n>5$ ) presented as arbitrary units (AU) if not stated otherwise were analyzed by Student's t test. Statistical significance is shown as ${ }^{*} \mathrm{p}<0.05$.

sured by levels of circulating thrombin-antithrombin complexes, $4 \mathrm{~h}$ after poly I:C injection (data not shown). To determine if thrombin contributes to antiviral responses after poly I:C injection, WT mice were fed with the thrombin inhibitor dabigatran etexilate ( $10 \mathrm{~g} / \mathrm{kg}$ chow) prior to poly I:C injection. The dabigatran etexilate treatment significantly reduced IFN $\gamma$, CXCL10, CCL5 and viperin but not IFN $\beta$ and IRF7 mRNA expression in the spleen $4 \mathrm{~h}$ after poly I:C injection of WT mice compared to placebo-treated WT mice (fig. 5a). As observed with PAR-1 ${ }^{-/-}$mice, splenic CXCL1 mRNA expression was significantly increased with dabigatran etexilate compared to placebo-fed WT mice (fig. 5a). Furthermore, CXCL10 protein levels in the spleen and plasma were significantly lower with dabigatran etexilate compared to in placebofed WT mice $4 \mathrm{~h}$ after poly I:C injection (fig. $5 \mathrm{~b}, \mathrm{c}$ ).

\section{PAR-1 Deficiency Is Associated with Reduced}

Antiviral Responses in the Spleen after CVB3 Infection

TLR3-dependent signaling in macrophages was shown to mediate protection after CVB4 infection [20].

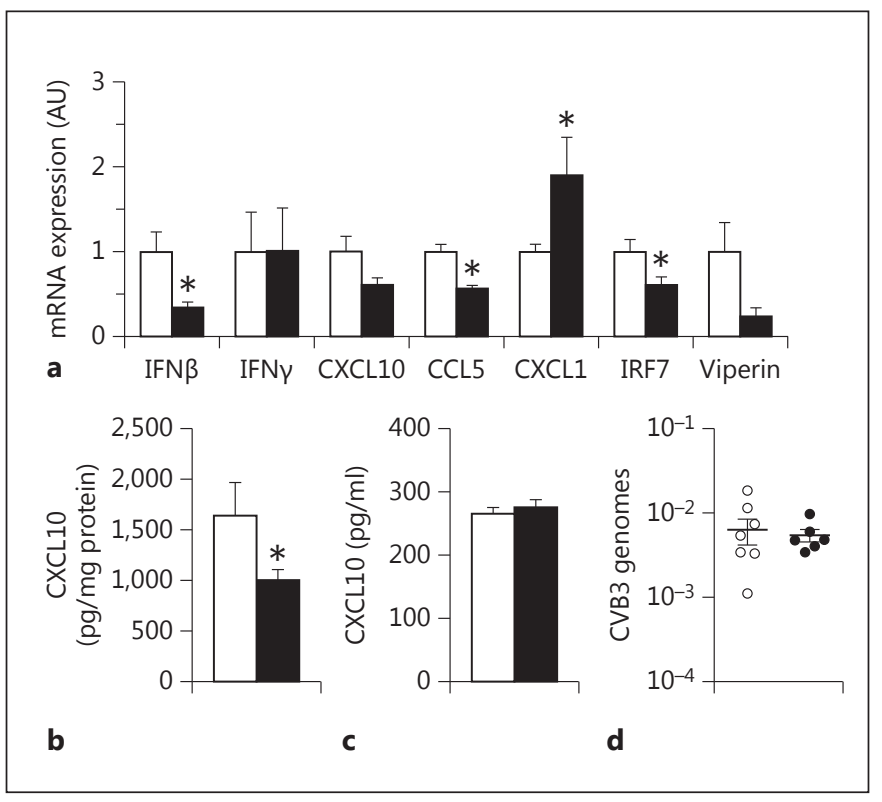

Fig. 6. PAR-1 deficiency is associated with altered immune responses in the spleen of CVB3-infected mice. WT (white bars) and PAR-1 ${ }^{-1-}$ (black bars) mice were infected with CVB3. a mRNA expression in the spleen 4 days after CVB3 infection. CXCL10 levels in the spleen (b) and plasma (c) 4 days after CVB3 infection. d CVB3 genome levels in the spleen. Data were normalized to Rpl4 mRNA (a) or $18 \mathrm{~S}$ (d) levels. Data (mean \pm SEM; $\mathrm{n}>5$ ) presented as arbitrary units (AU) if not stated otherwise were analyzed by Student's t test. Statistical significance is shown as ${ }^{*} \mathrm{p}<0.05$.

We found that PAR-1 deficiency was associated with reduced innate immune responses in the heart in CVB3 myocarditis [4]. In addition, CVB3 can infect and persist in the spleen and macrophages but its replication is highly suppressed [35-37]. WT and PAR- $1^{-/-}$mice were infected with CVB3 and the response in the spleen and plasma was analyzed. Consistent with our poly I:C data, PAR-1 $1^{-/-}$mice expressed significantly lower levels of IFN $\beta$, IRF7 and CCL 5 mRNA in the spleen compared to WT mice 4 days after CVB3 infection (fig. 6a). The expression of CXCL10 and viperin mRNA was also reduced in infected PAR-1 $1^{-/-}$mice spleens compared to WT mice spleens but this did not reach significance. There was no difference in IFN $\gamma$ expression between the genotypes. Furthermore, splenic CXCL1 mRNA increased significantly in PAR-1 $1^{-/}$mice spleens compared to infected WT mice spleens (fig. 6a). CXCL10 protein levels were significantly reduced in the spleen but not in the plasma (fig. 6b, c). As expected from the fact that CVB3 cannot replicate in macrophages, WT and PAR $-1^{-/-}$mice had similar levels of viral genomes 


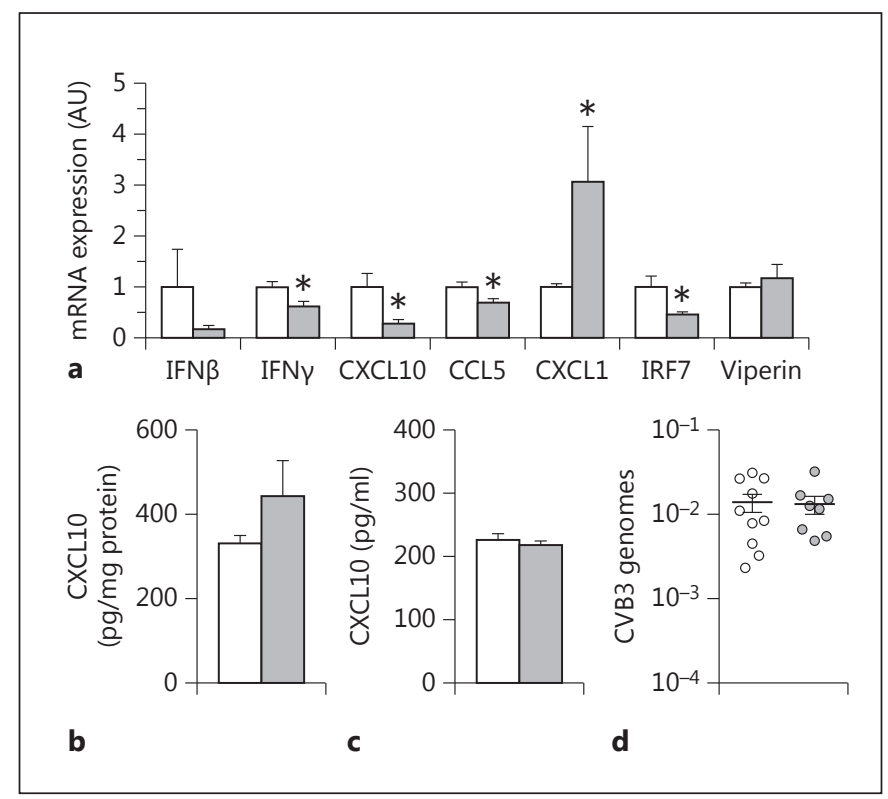

Fig. 7. Thrombin inhibition is associated with altered immune response in the spleen of CVB3-infected mice. WT mice were treated with dabigatran etexilate $(10 \mathrm{~g} / \mathrm{kg}$ chow, gray bars) or placebo (white bars) for 7 days prior to CVB3 infection. a Levels of mRNA expression in the spleen 4 days after CVB3 infection. CXCL10 levels in the spleen (b) and plasma (c) 4 days after CVB3 infection. d CVB3 genome levels in the spleen. Data were normalized to Rpl4 mRNA (a) or $18 \mathrm{~S}$ (d) levels. Data (mean \pm SEM; $\mathrm{n}>5$ ) presented as arbitrary units (AU) if not stated otherwise were analyzed by Student's t test. Statistical significance is shown as ${ }^{*} \mathrm{p}<0.05$.

(fig. 6d), indicating that the differences in the expression of antiviral genes was not due to different levels of virus.

\section{Inhibition of Thrombin Is Associated with a Reduced} Antiviral Response in the Spleen after CVB3 Infection

In our recent paper, we showed that thrombin inhibition increased CVB3 myocarditis in mice due to a decrease in the innate immune response [4]. In this study, we found that thrombin inhibition reduced the poly I:C induction of antiviral gene expression in the spleen. To determine if thrombin signaling contributes to the antiviral response in the spleen in CVB3 infected mice, we treated WT mice with dabigatran etexilate or placebo and then infected them with CVB3 and measured the antiviral response at 4 days. As expected, thrombin inhibition resulted in a significant reduction of the splenic IFN $\gamma$, CXCL10, CCL5 and IRF7 but not viperin mRNA expression (fig. 7a). IFN $\beta$ mRNA was reduced but this reduction did not reach significance (fig. 7a). However, we did not observe any differences in the CXCL10 levels in the spleen or plasma (fig. 7b, c). CXCL1 expression was increased in dabigatran-treated compared to placebo-treated mice 4 days after CVB3 infection (fig. 7a). The difference in the antiviral response in the spleen was due to the effect of thrombin on gene expression but not on virus load, since the spleens of infected dabigatran etexilate-treated and placebo-treated mice showed comparable CVB3 genome levels (fig. 7d).

\section{Discussion}

The immune and blood coagulation systems combine to combat infections $[17,18]$. dsRNA or poly I:C activation of TLR3 induces type I IFN innate immune responses as well as inducing TF expression and the activation of coagulation $[1,3,9,10,12,17]$. Here, we showed that PAR-1 deficiency is associated with a reduction in the poly I:C induction of IFN $\beta$ and CXCL10 expression in the spleen and plasma. This is likely due to reduced TLR3mediated responses in the immune cells, including splenic macrophages and DCs. Consistent with this notion, we found that PAR-1-deficient BMMs, BMDCs and splenocytes expressed lower levels of IFN $\beta$ and CXCL10 in vitro when stimulated with poly I:C and PAR-1 agonist compared to WT cells. Interestingly, we found that PAR-1 deficiency is associated with higher CXCL1 expression after poly I:C challenge. In addition, thrombin inhibition of WT mice produced similar results to in PAR- $1^{-/-}$mice, suggesting that thrombin-dependent PAR- 1 activation is important for the effective innate immune responses in the spleen. PAR- $1^{-/-}$mice treated with dabigatran etexilate and infected with CVB3 also showed a reduction in the antiviral response and an increase in CXCL1 compared with WT mice. Together, our results indicate that PAR-1 expression and the thrombin-PAR-1 pathway contribute to innate immune responses to poly I:C and viral infection.

TLR3 is activated by viral dsRNA reaching the endosomal compartment. This can occur when cells, such as macrophages and DCs, phagocytose dsRNA released into the extracellular space by dying virus-infected cells or when cells internalize viruses by receptor-mediated endocytosis [3]. Although normally restricted to the endosome, under certain conditions, TLR3 can be located on the cell surface $[3,4]$. TLR3 is expressed throughout the spleen [6]. In this study, we showed that PAR-1 is required for the maximal poly I:C induction of the IFN innate immune pathway in the spleen. The differences in 
gene expression observed in the spleen of PAR-1 $1^{-/-}$mice may, in part, explain the decreased levels of IFN $\beta$ and CXCL10 levels in the plasma of poly I:C-treated PAR$1^{-/-}$mice. This observation supports our recent findings and the proposed model of cooperative signaling between TLRs and PARs during infections $[4,5,9,10,19]$. The cells of the marginal zone of the spleen are the first to encounter the content of the blood, and may be the site where poly I:C is detected after i.p. injection [6]. Indeed, IFN $\beta$ was the major form of IFN produced in the red pulp and marginal zone of the spleen after poly I:C stimulation $[25,38]$. The difference in the splenic innate immune response after poly I:C injection appears to be due to cooperative signaling of PAR-1 and TLR3 on immune cells within the spleen, since isolated splenocytes exhibited a similar response when stimulated with poly I:C and PAR-1 agonist ex vivo. Furthermore, for some virus species, it has been shown that nonfibroblast-like cells, such as splenic marginal-zone macrophages and DCs, are the primary cells that respond with type I IFN and IFN-response gene expression after infection [39]. In addition, the depletion of phagocytic splenic macrophages and DCs of the marginal zone markedly reduced antiviral responses including the IFN-response genes, CXCL10 and CCL5 [32]. However, we cannot exclude the possibility of signaling from other dsRNA receptors, such as melanoma differentiation-associated gene 5 , which is also dependent on PAR-1 activation in vivo. This scenario does seem unlikely since we used nonliposome complexed, naked poly I:C, and $\mathrm{TLR}^{-1-}$ mice showed a dramatically reduced response to poly I:C (data not shown).

Surprisingly, we observed that the CXCL1 expression was negatively regulated by PAR-1 in splenocytes and in mice. CXCL1 is the murine homolog of human IL-8. PAR-1 activation reduced CXCL1 expression in splenocytes when cells were stimulated with poly I:C, and cells from PAR-1 $1^{-/}$mice expressed more CXCL1 after poly I:C stimulation. Furthermore, PAR-1 deficiency and thrombin inhibition increased CXCL1 expression in the spleen and plasma after poly I:C stimulation or CVB3 infection. Interestingly, it was shown that increased IRF3 activity was associated with higher levels of expression of IFN $\beta$, CXCL10 and IRF7 but lower levels of CXCL1/IL-8 and other proinflammatory cytokines in the microglia and astrocytes [40, 41]. This was due to protein kinase Bdependent inhibition of NF- $\kappa$ B. In addition, PAR-1 activation was shown to activate protein kinase $B$ [42]. Moreover, IFN $\beta$ was shown to suppress IL- 8 gene expression by modulating $\mathrm{NF}-\kappa \mathrm{B} \mathrm{p} 65$, and therefore reduced the binding to the $\kappa \mathrm{B}$ element within the IL- 8 promoter [43].
These findings are in line with our recently proposed hypothesis that PAR-1 activation inhibits the NF- $\kappa B$ pathway after TLR3 stimulation [9].

The TLR3-IFN pathway in immune cells, such as macrophages, was shown to limit viral replication of different viruses, such as $\mathrm{CVB}$, influenza, poliovirus, rhinovirus, herpes simplex and HIV [3, 7, 8, 20, 31]. Furthermore, we and other authors have shown that viral infections lead to increased TF expression in infected tissue and the activation of coagulation $[4,9-11,27]$. In addition, poly I:C injection leads to the activation of coagulation in mice [12]. More recently, it was shown that known PAR-1-activating proteases, such as MMP1 and MMP13, are highly expressed in respiratory syncytial virus-infected mouse lungs and infected human lung epithelial cells in vitro [44]. Here, we showed that thrombin is one of the possible activators of PAR-1 after poly I:C injections. Inhibition of thrombin during in vivo poly $\mathrm{I}: \mathrm{C}$ stimulation resulted in reduced IFN $\beta$ and IFN $\beta$-response gene expression in the spleen and plasma. However, the dabigatran etexilate effect was weaker than the effect observed with a global PAR-1 deficiency, which suggests that other PAR-1 activators such as MMPs may also be involved in the in vivo response to poly I:C. Furthermore, PAR-1 deficiency and thrombin inhibition led to reduced IFN responses in the spleen of mice 4 days after CVB3 infection. The observed reduction was not due to differences in virus-dependent thrombin-PAR-1 signaling, since the infected WT mice, $\mathrm{PAR}_{-1}{ }^{-/}$mice, placebo-treated mice and dabigatran etexilate-treated mice showed comparable splenic CVB3 genome levels.

Interestingly, PAR-1 and PAR-2 have opposite effects on TLR3-dependent induction of IFN $\beta$ expression $[9,10$, 45]. In contrast to PAR-1-deficient cells, PAR-2-deficient cells exhibit increased levels of IFN $\beta$ expression in response to poly I:C, and PAR $-2^{-/-}$mice are protected from CVB3 and influenza $\mathrm{A} / \mathrm{H} 1 \mathrm{~N} 1$ infection $[4,5,33]$. We speculate that the innate antiviral response is stimulated by host proteases, such as thrombin and MMP13, whereas viral proteases may activate PAR-2 in an attempt to dampen the antiviral response to the virus.

With regard to the potential clinical significance of our findings, it is possible that interference with the PAR-1 pathway from direct thrombin inhibitors, such as dabigatran etexilate, MMP13 inhibitors or direct PAR-1 inhibitors, may increase the risk and severity of viral infection as we showed in a mouse CVB3 myocarditis model [4]. However, the importance of PAR-1 signaling might be only applicable for an initial viral infection or in immunocompromised individuals. 
A different scenario may occur with virus infections that persist in the body and lead to chronic activation of the innate immune and clotting systems as observed in HIV [46, 47]. For instance, it was shown that, even under highly efficient antiretroviral therapy, an ongoing innate immune response and coagulation activation were associated with up to a 30 -fold increase in mortality in HIV patients compared to uninfected controls $[46,48]$. Despite undetectable virus levels, patients under antiretroviral therapy exhibit chronically elevated levels of type I IFNs, CXCL10 and coagulation markers, such as plasma TF and D-dimer [46-48]. In the case of a retroviral infection, anticoagulation and/or PAR-1 inhibition may reduce overall mortality of HIV-infected patients by directly affecting the activation of the coagulation system as well as dampen the activation of the coagulation-dependent immune system [47]. In line with our proposed mechanism, there are 2 ongoing clinical studies to use targeted anticoagulation therapy with the FXa inhibitor edoxaban (TACTICAL-HIV, NCT02339415) or the PAR-1 inhibitor vorapaxar (ADVICE, NCT02394730) in patients with HIV infection who are being successfully treated with combination antiretroviral therapy. Both studies will compare the safety and efficacy of either edoxaban or vorapaxar in reducing markers of cellular immune activation in HIV disease.

In summary, we show that PAR-1 activation contributes to poly I:C induction of TLR3/IFN responses in isolated macrophages and splenocytes and mice. Our results highlight the important crosstalk between the activation of coagulation and early innate antiviral responses in immune cells and the spleen in general.

\section{Acknowledgments}

We want to thank Ying Zhang and Wyeth Alexander for excellent technical assistance. The study was supported by grants from the Myocarditis Foundation (to S.A. and M.B.), the American Heart Association (14BGIA20380134, to S.A.), the Uehara Memorial Foundation (to K.T.) and the National Institutes of Health (HL119523 to N.M.).

\section{Disclosure Statement}

There were no competing interests.

\section{References}

1 Nan Y, Nan G, Zhang YJ: Interferon induction by RNA viruses and antagonism by viral pathogens. Viruses 2014;6:4999-5027.

2 Kato H, Takeuchi O, Sato S, Yoneyama M, Yamamoto M, Matsui K, Uematsu S, Jung A, Kawai T, Ishii KJ, Yamaguchi O, Otsu K, Tsujimura T, Koh CS, Reis e Sousa C, Matsuura Y, Fujita T, Akira S: Differential roles of MDA5 and RIG-I helicases in the recognition of RNA viruses. Nature 2006;441:101-105.

3 Zhang SY, Herman M, Ciancanelli MJ, Perez de Diego R, Sancho-Shimizu V, Abel L, Casanova JL: TLR3 immunity to infection in mice and humans. Curr Opin Immunol 2013;25: 19-33.

4 Antoniak S, Owens AP 3rd, Baunacke M, Williams JC, Lee RD, Weithauser A, Sheridan PA, Malz R, Luyendyk JP, Esserman DA, Trejo J, Kirchhofer D, Blaxall BC, Pawlinski R, Beck MA, Rauch U, Mackman N: PAR-1 contributes to the innate immune response during viral infection. J Clin Invest 2013;123:1310-1322.

5 Weithauser A, Bobbert P, Antoniak S, Bohm A, Rauch BH, Klingel K, Savvatis K, Kroemer HK, Tschope C, Stroux A, Zeichhardt H, Poller W, Mackman N, Schultheiss HP, Rauch U: Protease-activated receptor 2 regulates the innate immune response to viral infection in a CVB3-induced myocarditis. J Am Coll Cardiol 2013;62:1737-1745
6 McCartney S, Vermi W, Gilfillan S, Cella M, Murphy TL, Schreiber RD, Murphy KM, Colonna M: Distinct and complementary functions of MDA5 and TLR3 in poly(I:C)-mediated activation of mouse NK cells. J Exp Med 2009;206:2967-2976.

7 Sang M, Liu JB, Dai M, Wu JG, Ho WZ: Tolllike receptor 3 signaling inhibits simian immunodeficiency virus replication in macrophages from rhesus macaques. Antiviral Res 2014;112:103-112.

8 Zhou Y, Wang X, Liu M, Hu Q, Song L, Ye L, Zhou D, Ho W: A critical function of toll-like receptor-3 in the induction of anti-human immunodeficiency virus activities in macrophages. Immunology 2010;131:40-49.

9 Antoniak S, Mackman N: Multiple roles of the coagulation protease cascade during virus infection. Blood 2014;123:2605-2613.

10 Antoniak S, Mackman N: Coagulation, protease-activated receptors, and viral myocarditis. J Cardiovasc Transl Res 2014;7:203-211.

11 Antoniak S, Tatsumi K, Hisada Y, Milner JJ, Neidich SD, Shaver CM, Pawlinski R, Beck MA, Bastarache JA, Mackman N: Tissue factor deficiency increases alveolar hemorrhage and death in influenza A virus-infected mice. J Thromb Haemost 2016;14:1238-1248.
12 Shibamiya A, Hersemeyer K, Schmidt Woll T, Sedding D, Daniel JM, Bauer S, Koyama T, Preissner KT, Kanse SM: A key role for Tolllike receptor-3 in disrupting the hemostasis balance on endothelial cells. Blood 2009;113: 714-722.

13 Coughlin SR: Thrombin signalling and protease-activated receptors. Nature 2000;407: 258-264.

14 Jaffre F, Friedman AE, Hu Z, Mackman N, Blaxall BC: Beta-adrenergic receptor stimulation transactivates protease-activated receptor 1 via matrix metalloproteinase 13 in cardiac cells. Circulation 2012;125:2993-3003.

15 Austin KM, Covic L, Kuliopulos A: Matrix metalloproteases and PAR1 activation. Blood 2013;121:431-439.

16 Lopez ML, Soriano-Sarabia N, Bruges G, Marquez ME, Preissner KT, Schmitz ML, Hackstein H: Expression pattern of protease activated receptors in lymphoid cells. Cell Immunol 2014;288:47-52.

17 Shrivastava S, McVey JH, Dorling A: The interface between coagulation and immunity. Am J Transplant 2007;7:499-506.

18 Loof TG, Schmidt O, Herwald H, Theopold $\mathrm{U}$ : Coagulation systems of invertebrates and vertebrates and their roles in innate immunity: the same side of two coins? J Innate Immun 2011;3:34-40. 
19 Moretti S, Bellocchio S, Bonifazi P, Bozza S, Zelante T, Bistoni F, Romani L: The contribution of PARs to inflammation and immunity to fungi. Mucosal Immunol 2008;1:156-168.

20 Richer MJ, Lavallee DJ, Shanina I, Horwitz MS: Toll-like receptor 3 signaling on macrophages is required for survival following coxsackievirus B4 infection. PLoS One 2009; 4:e4127.

21 Darrow AL, Fung-Leung WP, Ye RD, Santulli RJ, Cheung WM, Derian CK, Burns CL, Damiano BP, Zhou L, Keenan CM, Peterson PA, Andrade-Gordon P: Biological consequences of thrombin receptor deficiency in mice. Thromb Haemost 1996;76:860-866.

22 Lee R, Williams JC, Mackman N: P2X7 regulation of macrophage tissue factor activity and microparticle generation. J Thromb Haemost 2012;10:1965-1967.

23 Gohlke PR, Williams JC, Vilen BJ, Dillon SR, Tisch R, Matsushima GK: The receptor tyrosine kinase MerTK regulates dendritic cell production of BAFF. Autoimmunity 2009;42: 183-197.

24 Sparkenbaugh EM, Chantrathammachart $\mathrm{P}$, Wang S, Jonas W, Kirchhofer D, Gailani D, Gruber A, Kasthuri R, Key NS, Mackman N, Pawlinski R: Excess of heme induces tissue factor-dependent activation of coagulation in mice. Haematologica 2015;100:308-314.

25 Ishikawa R, Biron CA: IFN induction and associated changes in splenic leukocyte distribution. J Immunol 1993;150:3713-3727.

26 Owens AP 3rd, Edwards TL, Antoniak S, Geddings JE, Jahangir E, Wei WQ, Denny JC, Boulaftali Y, Bergmeier W, Daugherty A, Sampson UK, Mackman N: Platelet inhibitors reduce rupture in a mouse model of established abdominal aortic aneurysm. Arterioscler Thromb Vasc Biol 2015;35:2032-2041.

27 Antoniak S, Boltzen U, Riad A, KallwellisOpara A, Rohde M, Dorner A, Tschope C, Noutsias M, Pauschinger M, Schultheiss HP, Rauch U: Viral myocarditis and coagulopathy: increased tissue factor expression and plasma thrombogenicity. J Mol Cell Cardiol 2008; $45: 118-126$

28 Antoniak S, Rojas M, Spring D, Bullard TA, Verrier ED, Blaxall BC, Mackman N, Pawlinski R: Protease-activated receptor 2 deficiency reduces cardiac ischemia/reperfusion injury. Arterioscler Thromb Vasc Biol 2010;30: 2136-2142.
29 Antoniak S, Sparkenbaugh EM, Tencati M, Rojas M, Mackman N, Pawlinski R: Protease activated receptor-2 contributes to heart failure. PLoS One 2013;8:e81733.

30 Davies LC, Jenkins SJ, Allen JE, Taylor PR: Tissue-resident macrophages. Nat Immunol 2013;14:986-995.

31 Oshiumi H, Okamoto M, Fujii K, Kawanishi T, Matsumoto M, Koike S, Seya T: The TLR3/ TICAM-1 pathway is mandatory for innate immune responses to poliovirus infection. J Immunol 2011;187:5320-5327.

32 Ciavarra RP, Taylor L, Greene AR, Yousefieh N, Horeth D, van Rooijen N, Steel C, Gregory B, Birkenbach M, Sekellick M: Impact of macrophage and dendritic cell subset elimination on antiviral immunity, viral clearance and production of type 1 interferon. Virology 2005;342:177-189.

33 Nhu QM, Shirey K, Teijaro JR, Farber DL, Netzel-Arnett S, Antalis TM, Fasano A, Vogel $\mathrm{SN}$ : Novel signaling interactions between proteinase-activated receptor 2 and Toll-like receptors in vitro and in vivo. Mucosal Immunol 2010;3:29-39.

34 Matsumoto T, Takahashi H, Shiva D, Kawanishi N, Kremenik MJ, Kato Y, Yano H: The reduction of voluntary physical activity after poly I:C injection is independent of the effect of poly I:C-induced interferon-beta in mice. Physiol Behav 2008;93:835-841.

35 Klingel K, Stephan S, Sauter M, Zell R, McManus BM, Bultmann B, Kandolf R: Pathogenesis of murine enterovirus myocarditis: virus dissemination and immune cell targets. J Virol 1996;70:8888-8895.

36 Lindner D, Li J, Savvatis K, Klingel K, Blankenberg S, Tschope C, Westermann D: Cardiac fibroblasts aggravate viral myocarditis: cell specific coxsackievirus B3 replication. Mediators Inflamm 2014;2014:519528.

37 Anderson DR, Wilson JE, Carthy CM, Yang D, Kandolf R, McManus BM: Direct interactions of coxsackievirus B3 with immune cells in the splenic compartment of mice susceptible or resistant to myocarditis. J Virol 1996; 70:4632-4645.
38 Scheu S, Dresing P, Locksley RM: Visualization of IFNbeta production by plasmacytoid versus conventional dendritic cells under specific stimulation conditions in vivo. Proc Natl Acad Sci USA 2008;105:20416-20421.

39 Barchet W, Cella M, Odermatt B, Asselin-Paturel C, Colonna M, Kalinke U: Virus-induced interferon alpha production by a dendritic cell subset in the absence of feedback signaling in vivo. J Exp Med 2002;195:507516.

40 Tarassishin L, Suh HS, Lee SC: Interferon regulatory factor 3 plays an anti-inflammatory role in microglia by activating the PI3K/Akt pathway. J Neuroinflamm 2011;8:187.

41 Park C, Lee S, Cho IH, Lee HK, Kim D, Choi SY, Oh SB, Park K, Kim JS, Lee SJ: TLR3-mediated signal induces proinflammatory cytokine and chemokine gene expression in astrocytes: differential signaling mechanisms of TLR3-induced IP-10 and IL-8 gene expression. Glia 2006;53:248-256.

42 Resendiz JC, Kroll MH, Lassila R: Proteaseactivated receptor-induced Akt activation regulation and possible function. J Thromb Haemost 2007;5:2484-2493.

43 Nozell S, Laver T, Patel K, Benveniste EN Mechanism of IFN-beta-mediated inhibition of IL-8 gene expression in astroglioma cells. J Immunol 2006;177:822-830.

44 Foronjy RF, Taggart CC, Dabo AJ, Weldon S, Cummins N, Geraghty P: Type-I interferons induce lung protease responses following respiratory syncytial virus infection via RIG-Ilike receptors. Mucosal Immunol 2015;8: 161-175.

45 Mackman N, Antoniak S: Roles of PAR1 and PAR2 in viral myocarditis. Thromb Res 2014; 133(suppl 1):S18-S20.

46 Tenorio AR, Zheng Y, Bosch RJ, Krishnan S, Rodriguez B, Hunt PW, Plants J, Seth A, Wilson CC, Deeks SG, Lederman MM, Landay AL: Soluble markers of inflammation and coagulation but not $\mathrm{T}$-cell activation predict non-AIDS-defining morbid events during suppressive antiretroviral treatment. J Infect Dis 2014;210:1248-1259.

47 Younas M, Psomas C, Reynes J, Corbeau P: Immune activation in the course of HIV-1 infection: causes, phenotypes and persistence under therapy. HIV Med 2016;17:89-105.

48 Funderburg NT, Lederman MM: Coagulation and morbidity in treated HIV infection. Thromb Res 2014;133(suppl 1):S21-S24. 